\title{
Prediction of Disposition Within 48 Hours of Hospital Admission Using Patient Mobility Scores
}

\author{
Daniel L Young, PT, DPT, PhD 1,2, Elizabeth Colantuoni, PhD², Lisa Aronson Friedman, ScM, Jason Seltzer, PT, DPT1, \\ Kelly Daley, PT, MBA', Bingqing Ye, MHA, MPA', Daniel J Brotman, MD5, Erik H Hoyer, MD ${ }^{1,5 \star}$
}

\begin{abstract}
${ }^{1}$ Department of Physical Medicine and Rehabilitation, Johns Hopkins University, Baltimore, Maryland; ${ }^{2}$ Department of Physical Therapy, University of Nevada Las Vegas, Las Vegas, Nevada; ${ }^{3}$ Department of Biostatistics, Johns Hopkins Bloomberg School of Public Health, Baltimore, Maryland; ${ }^{4}$ Division of Pulmonary and Critical Care Medicine, School of Medicine, Johns Hopkins University, Baltimore, Maryland; ${ }^{5}$ Division of General Internal Medicine, Johns Hopkins University, Baltimore, Maryland.
\end{abstract}

Delayed hospital discharges for patients needing rehabilitation in a postacute setting can exacerbate hospital-acquired mobility loss, prolong functional recovery, and increase costs. Systematic measurement of patient mobility by nurses early during hospitalization has the potential to help identify which patients are likely to be discharged to a postacute care facility versus home. To test the predictive ability of this approach, a machine learning classification tree method was applied retrospectively to a diverse sample of hospitalized patients $(\mathrm{N}=761)$ using training and validation sets. Compared with patients discharged to home, patients discharged to a postacute facility were older (median, 64 vs 56 years old) and had lower mobility scores at hospital admission (median, 32 vs 41 ). The final decision tree accurately classified the discharge location for $73 \%(95 \% \mathrm{Cl}, 67 \%-78 \%)$ of patients. This study emphasizes the value of systematically measuring mobility in the hospital and provides a simple decision tree to facilitate early discharge planning. Journal of Hospital Medicine 2020;15:540-543. () 2020 Society of Hospital Medicine

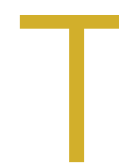

he loss of mobility during hospitalization is common and is an important reason why more than $40 \%$ of hospitalized Medicare patients require placement in a postacute facility. ${ }^{1,2}$ Discharge planning may be delayed when the medical team focuses on managing acute medical issues without recognizing a patient's rehabilitation needs until near the time of discharge. ${ }^{3}$ For patients who require rehabilitation in a postacute facility, delays in discharge can exacerbate hospital-acquired mobility loss and prolong functional recovery. ${ }^{2,4}$ In addition, even small increases in length of stay have substantial financial impact. ${ }^{5}$ Increased efficiency in the discharge process has the potential to reduce healthcare costs, facilitate patient recovery, and reduce delays for new admissions awaiting beds. ${ }^{6}$ For effective discharge planning, a proactive, patient-centered, interdisciplinary approach that considers patient mobility status is needed. ${ }^{3}$

Systematic measurement of patient mobility that extends beyond evaluations by physical therapists is not common practice but has the potential to facilitate early discharge planning. ${ }^{7.8}$ At our hospital, mobility assessment is performed routinely using a reliable and valid interdisciplinary assessment of

*Corresponding Author: Erik H Hoyer, MD; E-mail: ehoyer1@jhmi.edu; Telephone: 410-502-2441; Twitter: @HopkinsAMP.

Published online first December 18, 2019.

Received: July 19, 2019; Revised: September 11, 2019;

Accepted: September 20, 2019

๑) 2020 Society of Hospital Medicine DOI 10.12788/jhm.3332 mobility throughout the patient's entire hospitalization. ${ }^{9}$ We recently showed that nurse-recorded mobility status within the first 24 hours of hospitalization was associated with discharge disposition, but a prediction tool to help aid clinicians in the discharge planning process would be more useful. In this study, we evaluated the predictive ability of a patient's mobility score, obtained within 48 hours of hospital admission, to identify the need for postacute care in a diverse patient population.

\section{METHODS}

After receiving approval from the Johns Hopkins Institutional Review Board, we conducted analyses on a retrospective cohort of 821 admissions (777 unique patients admitted between January 1, 2017, and August 25, 2017) who were hospitalized for $\geq 72$ hours on two inpatient units (medical and neurological/neurosurgical) at The Johns Hopkins Hospital (JHH). These units were chosen to reduce the potential for both selection and measurement bias. First, these units manage a diverse patient population that is likely to generalize to a general hospital population. Second, the nursing staff on these units has the most accurate and consistent documentation compliance for our predictor variable.

\section{Mobility Measure}

The Activity Measure for Post Acute Care Inpatient Mobility Short Form (AM-PAC IMSF) is a measure of functional capacity. This short form is widely used and is nicknamed "6 clicks." It has questions for six mobility tasks, and each question is scored on a four-point Likert scale. ${ }^{9}$ Patients do not have to 
TABLE. Demographics for 805 admissions (761 unique patients) to The Johns Hopkins Hospital

\begin{tabular}{|c|c|c|c|}
\hline Characteristic & $\begin{array}{l}\text { All Patients } \\
\mathrm{N}=761\end{array}$ & $\begin{array}{c}\text { Discharged Home } \\
\quad n=469\end{array}$ & $\begin{array}{l}\text { Postacute Facility } \\
\quad n=292\end{array}$ \\
\hline Age (years), Median (IQR) & $59(46-69)$ & $56(43-67)$ & $64(54-72)$ \\
\hline Male, n (\%) & $405(53)$ & $253(54)$ & $152(52)$ \\
\hline \multicolumn{4}{|l|}{ Race $^{a}$} \\
\hline Other & $52(7)$ & $38(8)$ & $14(5)$ \\
\hline Characteristic & $\begin{array}{l}\text { All Admissions } \\
\qquad \mathrm{N}=\mathbf{8 0 5}\end{array}$ & $\begin{array}{c}\text { Discharged Home } \\
n=493\end{array}$ & $\begin{array}{c}\text { Postacute Facility } \\
n=312\end{array}$ \\
\hline \multicolumn{4}{|l|}{ Diagnosis } \\
\hline Medical/Other (\%) & $249(31)$ & $165(33)$ & $84(26)$ \\
\hline Hospital Admission AM-PAC IMSF Score, Median (IQR) & $38(32-42)$ & $41(36-44)$ & $32(26-38)$ \\
\hline Length of Stay (days), Median (IQR) & $7(5-10)$ & $6(4-8)$ & $8(6-13)$ \\
\hline
\end{tabular}

a Does not include the 38 patients of unknown race; 24 discharged home, 14 postacute care.

Abbreviations: AM-PAC, Activity Measure for PostAcute Care; IMSF, Inpatient Mobility Short Form; IQR, interquartile range.

attempt the tasks to be scored. Clinicians can score items using clinical judgment based on observation or discussion with the patient, family, or other clinicians. The interrater reliability is very good (Intraclass Correlation Coefficient $=.85-.99)^{9}$ and construct validity has been demonstrated for the inpatient hospital population (AM-PAC IMSF correlations with functional independence measure [FIM] $=.65$; Katz activities of daily living $[\mathrm{ADL}]=.80 ; 2$-minute walk $=.73 ;$ 5-times sit-to-stand $=$ -.69). ${ }^{9}$ At $\mathrm{JHH}$, the AM-PAC IMSF is scored at admission by nursing staff (>90\% documentation compliance on the units in this study); these admission scores were used.

\section{Outcome and Predictors}

Discharge location (postacute care facility vs home) was the primary outcome in this study, as recorded in a discrete field in the electronic medical record (EMR). To ensure the validity of this measure, we performed manual chart audits on a sample of patients $(n=300)$. It was confirmed that the measure entered in the discrete field in the EMR correctly identified the disposition (home vs postacute care facility) in all cases. The primary predictor was the lowest AM-PAC IMSF score obtained within 48 hours after hospital admission, reflecting the patient's capability to mobilize after hospital admission. Raw scores were converted to scale scores (0-100) for analysis. ${ }^{9}$ Additional predictors considered included age, sex, race, and primary diagnosis, all of which were readily available from the EMR at the time of hospital admission. We then grouped the primary diagnosis into the following categories using ICD-10 codes upon admission: Oncologic, Progressive Neurological, Sudden Onset Neurological, and Medical/Other.

\section{Statistical Analysis}

We constructed a classification tree, a machine learning approach, ${ }^{10}$ to predict discharge placement (postacute facility vs home) based on the patients' hospital admission characteristics and AM-PAC IMSF score. The prediction model was developed using the classification tree approach, as opposed to a logistic regression model. This approach allows for the inclusion of higher-order interactions (ie, interactions of more than two predictors) which would need to be explicitly specified otherwise and a priori we did not have strong evidence from prior studies to guide the model construction. The classification tree was constructed and evaluated by dividing our sample into a $70 \%$ training set and a $30 \%$ validation set using random sampling within key strata defined by age ( $<65$ vs $\geq 65$ years), gender, and quartile of the AM-PAC IMSF score. The classification tree was developed using the training set. Next, measures of predictive accuracy (ie, the proportion of correctly classified patients with placement in a postacute facility [sensitivity]) and the proportion of correctly classified patients not discharged to postacute care (ie, to home, specificity), were estimated by applying the validation set to the classification tree. The R statistical package rpart ${ }^{11}$ with procedure rpart was used to construct the classification tree using standard criteria for growing (Gini index $x^{10}$ ) and pruning (misclassification error estimated by leave-1-out cross-validation ${ }^{12}$ ) the tree. 


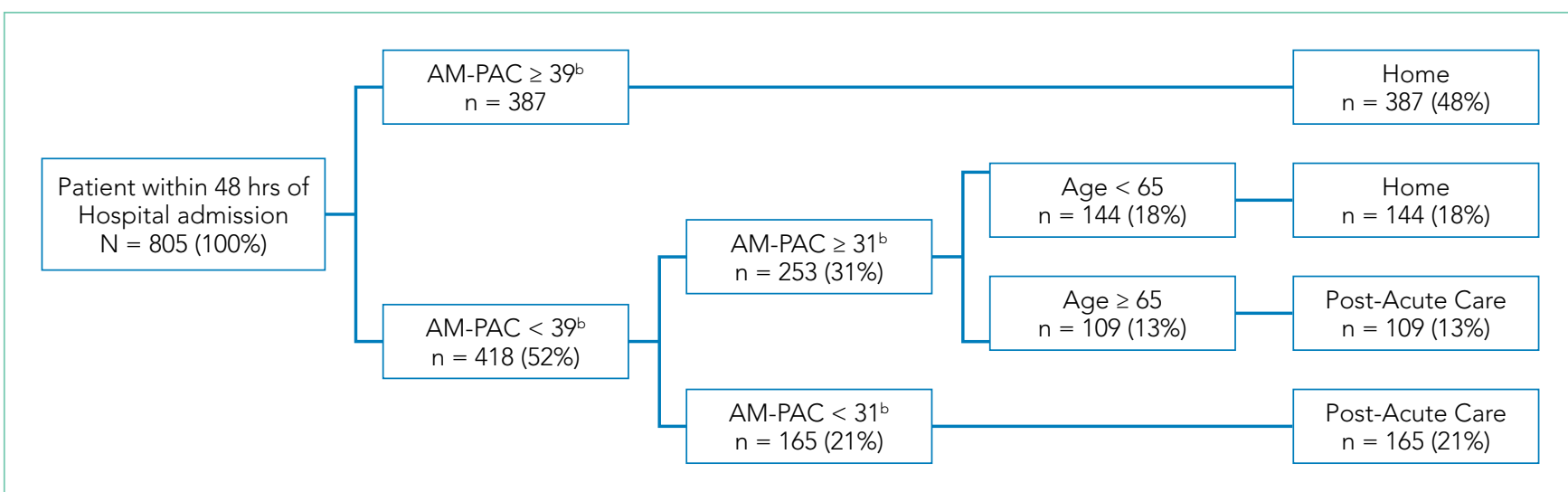

FIG. Decision Tree for Discharge Location-Postacute Care Facility versus Home

aDecision tree was created using a classification tree machine learning approach to predict discharge placement (postacute care facility vs home) considering the following variables: age, race, sex, diagnosis, and hospital admission AM-PAC Inpatient Mobility Short Form scores.

bAM-PAC IMSF Scale score values of 39 and 31 correspond with raw scores of 17 and 12, respectively

Abbreviations: AM-PAC, Activity Measure for PostAcute Care; IMSF, Inpatient Mobility Short Form.

\section{RESULTS}

Among the 821 admissions, 16 of 777 patients (2\%) died. Given the small number of deaths, we excluded these patients from the analysis. The table describes the characteristics of the 761 unique patients during each of their 805 admissions included in the analysis. Of these, 312 (39\%) were discharged to a postacute facility. Compared with patients discharged to home, patients discharged to a postacute facility were older (median, 64 vs 56 years), more likely to be admitted for a condition with sudden onset (eg, stroke, $36 \%$ vs $30 \%$ ), had lower AM-PAC IMSF scores at hospital admission (median, 32 vs 41), and longer lengths of stay (median, 8 vs 6 days). The figure displays the classification tree derived from the training set and the hospital admission characteristics described above, including the AM-PAC IMSF scores. The classification tree identified four distinct subsets of patients with the corresponding predicted discharge locations: (1) patients with AM-PAC IMSF scores 239 : discharged home, (2) patients with AM-PAC IMSF scores $\geq 31$ and $<39$ and who are $<65$ years of age: discharged home, (3) patients with AM-PAC IMSF scores $\geq 31$ and $<39$ and who are $\geq 65$ years of age: discharged to a postacute facility, and (4) patients with AM-PAC IMSF scores <31: discharged to a postacute facility. After applying this tree to the validation set, the specificity was $84 \%$ (95\% Cl: $78 \%-90 \%)$ and sensitivity was $58 \%$ (95\% Cl: $49 \%-68 \%$ ) for predicting discharge to a postacute facility, with an overall correct classification of $73 \%$ (95\% Cl: 67\%-78\%) of the discharge locations.

\section{DISCUSSION}

Improving the efficiency of hospital discharge planning is of great interest to hospital-based clinicians and administrators. Identifying patients early who are likely to need placement in a postacute facility is an important first step. Using a reliable and valid nursing assessment tool of patient mobility to help with discharge planning is an attractive and feasible approach. The literature on predicting disposition is very limited and has fo- cused primarily on patients with stroke or joint replacement. ${ }^{13,14}$ Previously, we used the same measure of mobility within 24 hours of admission to show an association with discharge disposition.? Here, we expanded upon that prior research to include mobility assessment within a 48-hour window from admission in a diverse patient population. Using a machine learning approach, we were able to predict $73 \%$ of hospital discharges correctly using only the patient's mobility score and age. Having tools such as this simple decision tree to identify discharge locations early in a patient's hospitalization has the potential to increase efficiency in the discharge planning process.

Despite being able to classify the discharge disposition correctly for most patients, our sensitivity for predicting postacute care need was low. There are likely other patient and system factors that could be collected near the time of hospital admission, such as the patient's prior level of function, the difference between function at baseline and admission, their prior living situation (eg, long-term care, home environment), social support, and hospital relationships with postacute care facilities that may help to improve the prediction of postacute care placement. ${ }^{15}$ We recommend that future research consider these and other potentially important predictors. However, the specificity was high enough that all patients who score positive merit evaluation for possible postacute care. While our patient sample was diverse, it did not focus on some patients who may be more likely to be discharged to a postacute facility, such as the geriatric population. This may be a potential limitation to our study and will require this tool to be tested in more patient groups. A final limitation is the grouping of all potential types of postacute care into one category since important differences exist between the care provided at skilled nursing facilities with or without rehabilitation and inpatient acute rehabilitation. Despite these limitations, this study emphasizes the value of a systematic mobility assessment and provides a simple decision tree to help providers begin early discharge planning by anticipating patient rehabilitation needs. 


\section{Acknowledgments}

The authors thank Christina Lin, MD, and Sophia Andrews, PT, DPT, for their assistance with data validation.

\section{References}

1. Greysen SR, Patel MS. Annals for hospitalists inpatient notes-bedrest is toxic-why mobility matters in the hospital. Ann Intern Med. 2018;169(2): HO2HO3. https://doi.org/10.7326/M18-1427

2. Greysen SR, Stijacic Cenzer I, Boscardin WJ, Covinsky KE. Functional impairment: an unmeasured marker of Medicare costs for postacute care of older adults. J Am Geriatr Soc. 2017;65(9):1996-2002. https://doi.org/10.1111/ jgs. 14955

3. Wong $\mathrm{EL}$, Yam CH, Cheung AW, et al. Barriers to effective discharge planning: a qualitative study investigating the perspectives of frontline healthcare professionals. BMC Health Serv Res. 2011;11(1):242. https://doi. org/10.1186/1472-6963-11-242

4. Greysen HM, Greysen SR. Mobility assessment in the hospital: what are the "next steps"? J Hosp Med. 2017;12(6):477-478. https://doi.org/10.12788/ jhm.2759

5. Lord RK, Mayhew CR, Korupolu R, et al. ICU early physical rehabilitation programs: financial modeling of cost savings. Crit Care Med. 2013;41(3):717-724 https://doi.org/10.1097/CCM.0b013e3182711de2

6. McDonagh MS, Smith DH, Goddard M. Measuring appropriate use of acute beds: a systematic review of methods and results. Health Policy. 2000;53(3):157-184. https://doi.org/10.1016/S0168-8510(00)00092-0

7. Hoyer EH, Young DL, Friedman LA, et al. Routine inpatient mobility assessment and hospital discharge planning. JAMA Intern Med. 2019;179(1):118120. https://doi.org/10.1001/jamainternmed.2018.5145

8. Brown CJ, Redden DT, Flood KL, Allman RM. The underrecognized epidem-
Disclosures: The authors certify that no party having a direct interest in the results of the research supporting this article has or will confer a benefit on them or on any organization with which they are associated.

ic of low mobility during hospitalization of older adults. J Am Geriatr Soc. 2009;57(9):1660-1665. https://doi.org/10.1111/j.1532-5415.2009.02393.x

9. Hoyer EH, Young DL, Klein LM, et al. Toward a common language for measuring patient mobility in the hospital: reliability and construct validity of interprofessional mobility measures. Phys Ther. 2018;98(2):133-142. https://doi. org/10.1093/ptj/pzx110

10. Breiman L, Friedman J, Olshen R, Stone C. Classification and Regression Trees. Wadsworth; 1984

11. Therneau T, Atkinson B. rpart: recursive partitioning and regression trees. $R$ package version. 2018;4:1-13. https://CRAN.R-project.org/package=rpart

12. Friedman J, Hastie T, Tibshirani R. The Elements of Statistical Learning. Springer; 2001.

13. Stein J, Bettger JP, Sicklick A, Hedeman R, Magdon-Ismail Z, Schwamm LH. Use of a standardized assessment to predict rehabilitation care after acute stroke. Arch Phys Med Rehabil. 2015;96(2):210-217. https://doi.org/10.1016/j. apmr.2014.07.403

14. Gholson JJ, Pugely AJ, Bedard NA, Duchman KR, Anthony CA, Callaghan JJ. Can we predict discharge status after total joint arthroplasty? A calculator to predict home discharge. J Arthroplasty. 2016;31(12):2705-2709. https://doi. org/10.1016/j.arth.2016.08.010

15. Zimmermann BM, Koné I, Rost M, Leu A, Wangmo T, Elger BS. Factors associated with post-acute discharge location after hospital stay: a cross-sectional study from a Swiss hospital. BMC Health Serv Res. 2019;19(1):289. https:// doi.org/10.1186/s12913-019-4101-6 